\title{
Personalizing Energy Expenditure Estimation Using a Cardiorespiratory Fitness Predicate
}

\author{
Marco Altini ${ }^{1,2}$ and Julien Penders ${ }^{1}$ \\ ${ }^{1}$ Holst Centre/imec The Netherlands \\ ${ }^{2}$ ACTLab, Eindhoven University of Technology \\ Eindhoven, The Netherlands \\ Email: altini.marco@gmail.com
}

\author{
Oliver $\mathrm{Amft}^{2}$ \\ ${ }^{2}$ ACTLab, Eindhoven University of Technology \\ Eindhoven, The Netherlands
}

\begin{abstract}
Accurate Energy Expenditure (EE) estimation is key in understanding how behavior and daily physical activity (PA) patterns affect health, especially in today's sedentary society. Wearable accelerometers (ACC) and heart rate (HR) sensors have been widely used to monitor physical activity and estimate EE. However, current EE estimation algorithms have not taken into account a person's cardiorespiratory fitness (CRF), even though CRF is the main cause of inter-individual variation in HR during exercise. In this paper we propose a new algorithm, which is able to significantly reduce EE estimate error and inter-individual variability, by automatically modeling $C R F$, without requiring users to perform specific fitness tests. Results show a decrease in Root Mean Square Error (RMSE) between 28 and 33\% for walking, running and biking activities, compared to state of the art activity-specific EE algorithms combining ACC and HR.
\end{abstract}

\section{INTRODUCTION}

Accurate monitoring of physical activity (PA) patterns (type, intensity, frequency and duration) is key in unveiling the relation between aspects of human behavior and health status [1]. Especially in today's western world, where computer based work is predominant, it is important to understand the relation between aspects of PA and diseases such as obesity and diabetes, diseases that started affecting our society only in the recent past. To determine the links between aspects of behavior related to physical activity and health status, accurate quantification and assessment of habitual physical activity in ambulatory settings is essential. New technologies, seamlessly integrated in everyone's life, able to monitor objectively and non-invasively our behavior, can provide unprecedented insights on these links. Among the technologies used to objectively monitor PA, accelerometers (ACC) and heart rate (HR) monitors are the most widespread [2-9]. For ACC, the rationale behind their adoption is the linear relation between motion close to the body's center of mass, and energy expenditure (EE). On the other hand, HR shows a strong correlation with $\mathrm{EE}$, due to the relation between oxygen consumption, HR and EE. Main limitations of these technologies are the inability of single accelerometers close to the body's center of mass to detect low and upper body motion, and the low accuracy of HR monitors during sedentary behavior, as well as the need for individual calibration. Some of these issues have been tackled by developing activity-specific EE algorithms [5-9].

Activity-specific EE algorithms subdivide the estimation process into two steps. First, the activity performed by the user is determined. Secondly, an activity-specific equation is applied to estimate EE. By first recognizing the activity, the lack of agreement between ACC and EE when no whole body motion is involved (e.g. while biking) can be accounted for by for example assigning static EE values to such activities. Normally, activity-specific equations use ACC features and anthropometric characteristics to predict EE. Some activityspecific algorithms use equations where HR is included as well [5-6], showing consistent improvement compared to ACC alone [5]. By adopting an activity-specific approach, some HR limitations can be easily overcome. Issues due to the weak relation between HR and EE during sedentary time, where HR can be affected by artifacts due to emotions and stress, can be avoided by including HR only in some activity-specific equations (e.g. when moderate to vigorous PA is performed). The need for individual calibration of HR-based algorithms is motivated by the substantial inter-individual differences in the relation between HR and EE. During moderate to vigorous PA, differences in HR between individuals performing the same activity are mainly due to cardiorespiratory fitness (CRF). CRF, is not only the main cause of inter-individual variability, but also inversely related with several health outcomes, such as cardiovascular disease and coronary artery disease, being one of the most important health markers [10]. Combined with activity-specific algorithms, information on CRF could provide more accurate EE estimation. Nevertheless, algorithms in the past tackled CRF-related variance only by means of individual calibration [2], and no algorithm includes information on CRF in the EE estimation equations. For many practical applications individual calibration is not feasible since it would require every user to perform a calibration test.

In this paper, we present a new activity-specific EE algorithm that incorporates CRF-related variance by normalizing HR. The HR normalization is performed by estimating walking speed and activities, and integrating anthropometric information. In particular, the following contributions are made:

1) We detail the HR normalization procedure that, based on activities carried out during daily life (rest, walking at different speeds), can automatically estimate CRF-related variance. Thus, our approach does not require users to do specific fitness tests to estimate CRF.

2) We compare EE estimation performance of a standard current state of the art activity-specific algorithm with our personalizing version considering individual CRF. For this purpose, we used a dataset including 44 activities recorded with 29 subjects. 
This paper is structured as follows. Related work and the relation between CRF, HR and EE are discussed in Sections II and III. Section IV introduces our approach to CRF estimation and HR normalization. The implementation of our approach is described in Section V, while the measurement setup and data collection process can be found in Section VI. Results and conclusions are presented in Sections VII and VIII.

\section{RELATED WORK}

\section{A. Epidemiological Research}

Accelerometer and HR monitors are the most commonly used single sensor devices in epidemiological studies $[2,3,4,14]$. ACC use activity counts, a unit-less measure representative of whole body motion, as independent variable in the linear regression model developed to predict EE [3]. Shortcomings of single regression models are; $a$ ) the accuracy of the monitor is highly dependent on the activities used to develop the linear model, $b$ ) a single linear model does not fit all the activities, since the slope and intercept of the regression model change based on the activity performed while data is collected. As a result, even when activity counts are representative of $\mathrm{EE}$, the output can be misleading.

HR monitors suffer from different problems. First, HR monitors are typically inaccurate during sedentary behavior, given the fact that HR is also affected by non-activity related factors, such as stress and emotions [4]. Artifacts at rest were tackled by means of the so called HR-flex point, a point above which EE is estimated using an activity-model, while below which EE is estimated using a rest value or a sedentarymodel [4]. Basically, a first version of today's activity-specific algorithms [5-9]. Secondly, HR monitors need individual calibration to perform accurately [2]. The high correlation between $\mathrm{HR}$ and EE within one individual, which motivated researchers in using HR monitors to estimate EE since the 80s, is indeed peculiar of a specific individual, and changes substantially between subjects. Even the HR-flex point, is often determined specifically for one individual, by for example averaging the $\mathrm{HR}$ at rest and the HR while walking at a certain speed.

\section{B. Activity-specific EE estimation}

The latest monitors extended approaches based on simple linear regression models performing activity recognition over a predefined set of activities, and then applying different methods to predict EE [5-9], based on the activity. The principle behind activity recognition as a first step in EE estimation is that the slope and intercept of the regression models change based on the activity performed. One approach [9] is to apply a different regression equation for each activity classified. The regression models typically use ACC features and anthropometric characteristics as independent variables. Another approach is to assign static values (e.g. Metabolic Equivalents (METs) from the compendium on physical activities) to each one of the clusters of activities. Assigning static values showed limitations during moderate to vigorous activities in a recent comparison between activity-specific models, since static values cannot capture intra-individual differences in $\mathrm{EE}$ [5]. Intra-individual differences in $\mathrm{EE}$ for an activity are caused by the fact that moderate to vigorous activities can be carried out at different intensities (e.g. walking at different speeds), resulting in different levels of EE. Activity-specific linear regression models require $\mathrm{ACC}$ and $\mathrm{HR}$ features to capture these differences [5].

Some authors included HR features as well in the activityspecific linear models. In [6], a multi-sensor system composed of three accelerometers was developed. The authors extended the static approach of [8], developing a custom MET table, which takes into account anthropometric variables, as well as the HR at rest, to predict EE. In [7] HR and ACC were combined as well. The system consisted of three sensors, two accelerometers and a HR belt, and could classify seven types of activities. Inter-individual differences in HR were not taken into account.

Activity-specific multiple linear regression models combining ACC and HR features showed consistent improvements in EE estimation accuracy compared to algorithms using static or ACC-only features [5]. However, inter-individual differences in HR due to CRF are not tackled by any activity-specific algorithm. One approach used to reduce inter-individual differences in HR during daily life, was proposed in [3]. The authors use the Heart Rate above Rest (HRaR), instead of the $\mathrm{HR}$, as a predictor for their linear models. Using the $\mathrm{HRaR}$ does bring each subject to the same baseline, but it introduces a simple offset, which is unable to capture how HR evolves during physical exercise, as a result of differences in CRF.

\section{CRF Estimation}

Even though the effects of CRF on HR are widely recognized, no algorithm up to date includes or models CRF to estimate EE. On the other hand, different groups proposed methods and algorithms to measure and estimate CRF alone $[13,16]$. CRF is typically measured by means of a maximal oxygen uptake test. Maximal oxygen uptake $\left(\mathrm{VO}_{2} \max \right)$ is widely accepted as the single best measure of cardiovascular fitness and maximal aerobic power. Tests measuring $\mathrm{VO}_{2}$ max can be dangerous in individuals who are not considered normal healthy subjects, as any problems with the respiratory and cardiovascular systems will be greatly exacerbated. Thus, many protocols for estimating $\mathrm{VO}_{2}$ max have been developed for those for whom a traditional $\mathrm{VO}_{2}$ max test would be too risky $[13,16]$. Sub-maximal $\mathrm{VO}_{2}$ max tests generally are similar to a $\mathrm{VO}_{2}$ max test, but do not reach the maximum of the respiratory and cardiovascular systems. On the other hand, non-exercise $\mathrm{VO}_{2}$ max estimation uses information about the person's anthropometric characteristics, activity level (derived with questionnaires), and $\mathrm{HR}$ at rest features to estimate CRF. Often, the predicted maximal HR is used as well. The shortcoming of this approach is that maximal HR is typically predicted using age only, and HR at rest is weakly related to CRF. Higher accuracy was shown by sub-maximal tests involving actual exercise, for example biking or running at sub-maximal rates.

Even though sub-maximal tests are less dangerous and showed good accuracy in past research [13], they are still affected by some limitations; $a$ ) a specific test is required to determine CRF, $b$ ) the specific test should be re-performed every time CRF needs to be assessed, $c$ ) in the context of EE estimation, it is not clear how to include information about CRF. 

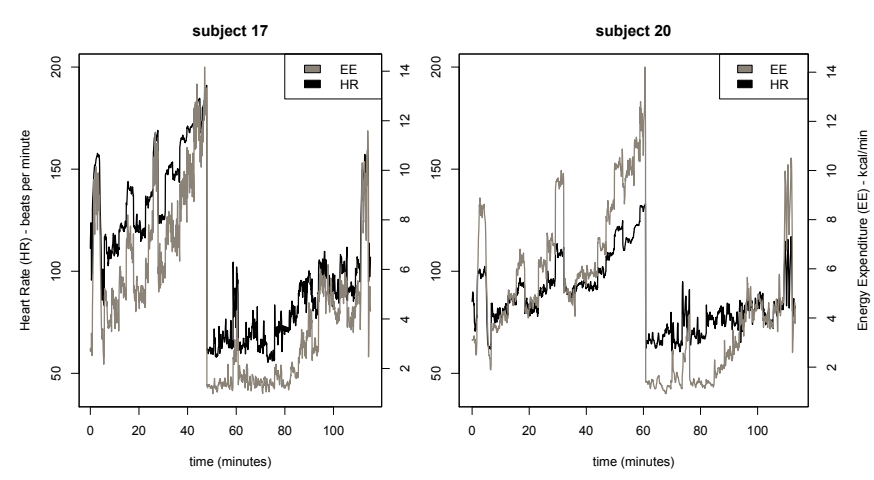

Fig. 1. Relation between EE and HR in different subjects during a sequence of different PAs. Individual correlations between EE and HR are 0.97 and 0.96 respectively. The absolute EE levels are similar due to similar body weight. HR differs significantly between the subjects during moderate to vigorous activities.

\section{Fitness And HeART Responses}

This section covers more in detail the relation between CRF, HR and EE, which motivates our approach to personalized EE estimation. The main cause of differences in the HREE relation during activity is CRF. An individual with higher CRF (i.e. more fit), will have a lower HR during exercise, compared to an individual with low CRF. Fig. 1 shows the relation between $\mathrm{HR}$ and $\mathrm{EE}$ for two subjects during a series of intense and sedentary activities. Individual correlations between HR and EE are above 0.96 for both of them. The figure shows clearly that for two subjects with similar body weight (subject 17, body weight: $71.2 \mathrm{~kg}$, and subject 20,72 $\mathrm{kg}$ ), $\mathrm{EE}$ is almost the same, while HR is very different, due to higher fitness level of subject 20 (subject 17 is inactive while subject 20 is a trained runner). Since EE is derived from HR, typically by means of a linear model, estimating EE from HR during exercise results into substantially high over and under-estimations. By individually calibrating the system, the relation between $\mathrm{HR}$ and EE becomes peculiar for an individual, since it is derived specifically for him/her, and not using data collected on a different sample of the population. Unfortunately, individual calibration is not practically feasible since it requires each new user to perform lab tests in supervised settings, using expensive devices such as an indirect calorimeter. Thus, alternative methods to tackle the problem are needed to objectively and accurately estimate EE at the individual level, and not only as group averages.

\section{APPROACH}

This section covers our approach to CRF estimation and its integration into $\mathrm{EE}$ multiple linear regression models, necessary to reduce EE estimation error due to inter-individual differences in the relation between HR and EE. To this aim, we developed the concept of automatic Heart Rate Feature Normalization. We propose the following steps to estimate EE using HR normalized by level of CRF:

a) Build a model to derive a normalization factor automatically during daily life, without requiring specific tests.

b) Use the normalization factor to normalize HR.

c) Use the normalized HR as predictor in activity-specific EE estimation equations.

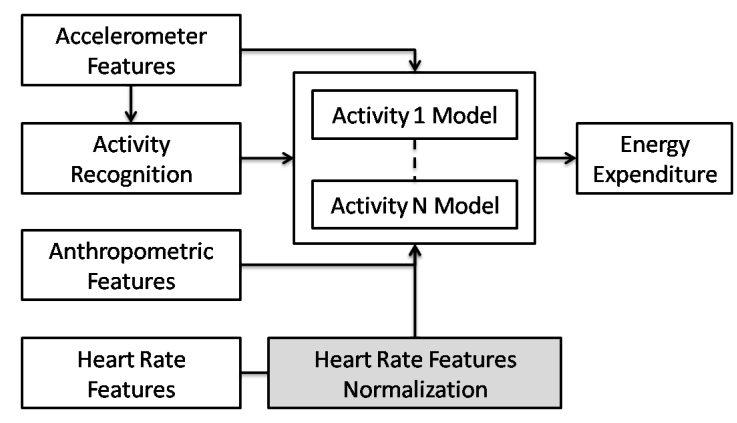

Fig. 2. Architecture of an activity-specific EE estimation system. ACC features are used to recognize an activity, and to estimate EE for each model, together with anthropometric characteristics and the normalized HR. The Heart Rate Features Normalization block, shown in gray, is included in our model to remove confounding effects in the relation between HR and EE, due to $\mathrm{CRF}$.

When normalizing HR, we are not only interested in the maximal HR an individual can reach, but in the HR the different individuals would reach when performing the same activity, at the same workload. We hypothesize that this HR at a constant workload is representative of CRF, and can be used to normalize HR. We selected running at $10 \mathrm{~km} / \mathrm{h}$ as the constant workload. Thus, our HR normalization factor is the $\mathrm{HR}$ while running at $10 \mathrm{~km} / \mathrm{h}$. Running at maximum 10 (for females) to 12 (for males) $\mathrm{km} / \mathrm{h}$, together with anthropometric characteristics, could explain $88 \%$ of the variance in $\mathrm{VO}_{2}$ max in past research on $\mathrm{VO}_{2}$ max estimation [13], showing that it is a normalization factor that well represents CRF. We implemented a system able to derive the CRF-related normalization factor automatically during daily life, without requiring specific tests (a). In this way the algorithm is able to selfadapt and learn from its user, without requiring any individual calibration to estimate CRF or EE. Once a normalization factor for an individual has been automatically determined, it is used to normalize HR (b). The resulting normalized HR is free of confounding effects due to CRF. As a last step, the normalized HR is used instead of the HR as a predictor for the activityspecific EE estimation equations (c), as shown in Fig. 2. The new predictor better represents the relation between HR and $\mathrm{EE}$, since $\mathrm{HR}$ is not affected by CRF.

TABLE I. DISTRIBUTION OF THE ACTIVITIES INTO THE SIX CLUSTERS USED FOR ACTIVITY RECOGNITION.

\begin{tabular}{|c|l|}
\hline Cluster name & Original activities \\
\hline Lying & Lying down resting \\
\hline Sedentary & $\begin{array}{l}\text { Sitting resting, sitting stretching, standing } \\
\text { stretching, desk work, reading, writing, } \\
\text { working on a PC, watching TV, sitting } \\
\text { fidgeting legs, standing still }\end{array}$ \\
\hline Dynamic & $\begin{array}{l}\text { Stacking groceries, washing dishes, cook- } \\
\text { ing, folding clothes, cleaning and scrub- } \\
\text { bing, washing windows, sweeping, vacu- } \\
\text { uming }\end{array}$ \\
\hline Walking & $\begin{array}{l}\text { Self-paced, self-paced carrying books, } \\
\text { stairs up and down, treadmill (flat: }\end{array}$ \\
& $3,4,5,6 \mathrm{~km} / \mathrm{h}, 4 \mathrm{~km} / \mathrm{h}$ carrying weights, \\
incline: $3,5 \mathrm{~km} / \mathrm{h}, 5,10 \%))$
\end{tabular}




\section{METHODS}

We considered ACC and HR data to implement all components of our approach, including activity recognition, HR normalization factor estimation, and EE estimation. This section details the components further. More details on participants, sensor device and experimental protocol can be found in Section VI.

\section{A. Activity Recognition}

We implemented an activity recognition algorithm to classify the following clusters of activities (see Table I): lying, sedentary, dynamic, walking, running and biking. We selected Support Vector Machines (SVMs) as classifier, and the following features: mean absolute value of the band-passed signa, variance, standard deviation, main frequency peak, amplitude of the main frequency peak and high frequency band signal power. See Sections V-E and V-F for details on the feature extraction and selection processes. For the SVM, a polynomial kernel with degree 5 was used $(\lambda=10, C=1)$. Activity recognition is used for EE estimation (all six clusters), and as part of the automatic HR normalization system (lying and walking only).

\section{B. Automatic HR Normalization System}

We extended the architecture of activity-specific EE estimation algorithms (Fig. 2), by including the extra Heart Rate Features Normalization block. The block is detailed in Fig. 3 , where all the components necessary to derive the normalization factor automatically, are listed. In order to provide automated and non-invasive CRF estimation, we estimate the normalization factor using activities of daily living only, and their associated HR. More specifically, the Heart Rate Features Normalization block uses the HR while resting and walking at different speeds as predictors for the HR normalization factor, together with anthropometric characteristics. Thus, in addition to the activity recognition algorithm, the automatic HR normalization system includes two more components; 1) a walking speed estimator, 2) and a normalization factor estimator. The next sections covers the components in detail.

1) Walking Speed Estimator: The walking speed estimator is a multiple linear regression model (see Table II) which predicts walking speed using as features the individual's height and the following ACC features: main frequency peak on the $X$ axis (FFTpeakXf), mean absolute value of the band-passed signal (or Motion Intensity, MI), sum of the variance on the three axis (Var), inter-quartile range on the $X$ and $Y$ axis (IQRX and $I Q R Y)$ and high frequency band signal power on the $X$ and $Z$ axis (HPowX and HPowZ).

TABLE II. WALKING SPEed Model $\left(R^{2} 0.94\right)$.

\begin{tabular}{|c|c|}
\hline Variable & Coefficient \\
\hline Intercept & -1.28 \\
Height & 0.015 \\
MI & 11.37 \\
Var & -2.41 \\
IQRX & 1.79 \\
IQRY & -2.96 \\
HPowX & -0.00079 \\
HPowZ & -0.00084 \\
FFTpeakXf & -0.088 \\
\hline
\end{tabular}

TABLE III. HEART RATE Normalization FACtor Estimation MODEL $\left(R^{2} 0.87\right)$.

\begin{tabular}{|c|c|}
\hline Variable & Coefficient \\
\hline Intercept & 66.91 \\
HR at rest & 0.29 \\
HR $4 \mathrm{~km} / \mathrm{h}$ & 1.58 \\
HR $5 \mathrm{~km} / \mathrm{h}$ & -2.80 \\
HR $6 \mathrm{~km} / \mathrm{h}$ & 2.18 \\
Height & -0.17 \\
Age & -0.23 \\
\hline
\end{tabular}

2) Heart Rate Normalization Factor Estimator: A multiple linear regression model (see Table III) is built to predict the normalization factor (i.e. an individual's HR while running at $10 \mathrm{~km} / \mathrm{h}$ ) using activities of daily living only. The best model (see Section VII) relies on HR while lying down resting and while walking at 4, 5 and $6 \mathrm{~km} / \mathrm{h}$, together with the individual height and age, as independent variables.

\section{HR Normalized}

Actual HR measurements are finally used after applying the HR normalization factor, derived with the normalization factor estimator, using the simple ratio:

$$
\text { NormalizedHR }=\frac{\text { Current } H R}{\text { Normalizationfactor }}
$$

\section{Personalized activity-specific EE estimation}

Following the methodology applied in current state of the art EE estimation algorithm, EE is estimated by first classifying the activity performed, by means of ACC features, and then applying an activity-specific EE linear regression model. The activity-specific EE linear models use anthropometric characteristics, ACC and HR features. Thus, we developed six multiple linear regression models, one for each cluster of activities (see Table IV). Activity-specific ACC features for each model were selected using linear forward selection, in order to model intra-individual differences in EE. Normalized HR was used as a feature for the moderate to vigorous clusters (dynamic, walking, running and biking). The final feature set includes Resting Metabolic Rate (RMR, computed with anthropometric variables only, according to the Harris-Benedict formula), motion intensity $(M I)$, standard deviation $(S T D)$, median (MED), main frequency peak (FFTpeax $\left.{ }_{f}\right)$ and its amplitude $\left(F F T p e a k_{a}\right)$, body weight $(B W)$ and Normalized Heart Rate (HRNorm).

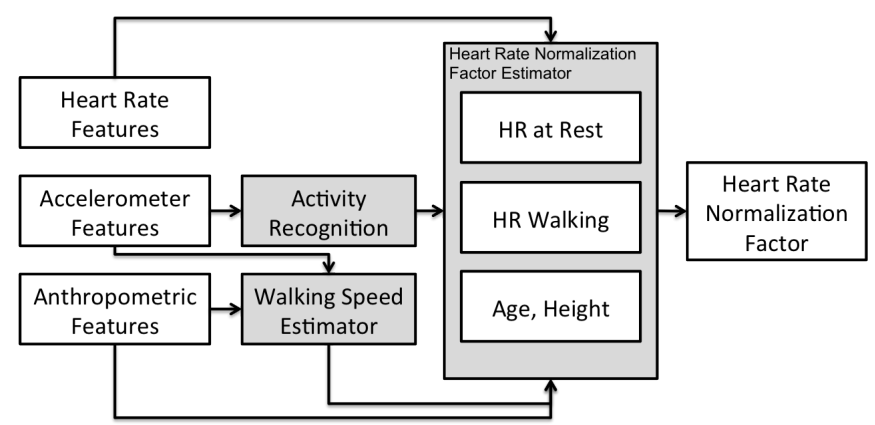

Fig. 3. Components of the automatic HR normalization system used in this work to derive the normalization factor automatically. 
TABLE IV. ACTIVITY-SPECIFIC EE LINEAR MODELS.

\begin{tabular}{|c|c|}
\hline Cluster & Model \\
\hline Lying & $\begin{array}{l}0.49+0.00068 \text { RMR }-29.66 M I x+ \\
9.78 S T D x+0.11 M E D x+0.68 M E D y\end{array}$ \\
\hline Sedentary & $\begin{array}{l}0.31+0.00061 R M R+8.42 M I x+ \\
11.12 \text { MIy }-2.37 M I z+2.9 \text { STD }+ \\
2.48 \text { STDy }+0.47 \text { MEDy }-0.14 \text { MEDz+ } \\
0.05 \text { FFTpeakYa }\end{array}$ \\
\hline Dynamic & $\begin{array}{l}-3.43+5.95 \text { HRNorm }+0.035 B W+ \\
7.65 \text { MIy }+8.59 \text { MItot }+4.80 \text { STD } x\end{array}$ \\
\hline Walking & $\begin{array}{l}-9.00+15.07 \text { HRNorm }+0.056 B W+ \\
3.91 \text { STD }\end{array}$ \\
\hline Biking & $\begin{array}{l}-10.58+0.0029 R M R+16.75 \text { HRNorm }- \\
37.66 \text { MIx }+14.23 \text { MIy }-54.37 \text { VARy }+ \\
26.22 \text { STD } x\end{array}$ \\
\hline Running & $\begin{array}{l}-8.73+11.50 \text { HRNorm }+0.12 B W+ \\
13.99 M I y-5.28 \text { STD }+4.16 \text { MED }- \\
3.70 \text { MEDz-1.33 FFTpeakXf }\end{array}$ \\
\hline
\end{tabular}

\section{E. Feature Extraction}

ACC and HR features were used to derive activity recognition, walking speed, CRF (normalization factor) estimation and EE estimation linear models. ACC data from the three axes were segmented in 4 second windows, band-pass (BP) filtered between 0.1 and $10 \mathrm{~Hz}$, to isolate the dynamic component caused by body motion, and low-pass (LP) filtered at $1 \mathrm{~Hz}$, to isolate the static component, due to gravity. We selected a time window of $4 s$, since it is short enough to detect changes in postures even for short breaks in sedentary time, and long enough to capture the repetitive patterns of activities, such as walking or running. Time and frequency features were extracted from each window over the three axes of the LP and BP signal. Time features included mean, mean of the absolute signal, magnitude, mean distance between axes, skewness, kurtosis, variance, standard deviation, coefficient of variation, range, min, max, correlation, inter-quartiles range, median and zero crossing rate. Frequency features included: spectral energy, entropy, low frequency band signal power (0.1 - $0.75 \mathrm{~Hz})$, high frequency band signal power $(0.75-10$ $\mathrm{Hz}$ ), frequency and amplitude of the FFT coefficients. These features were selected due to high accuracy showed in past research [5-9]. The mean $H R$ was extracted from R-R intervals, computed over 15 seconds windows. R-R intervals features were not included in the activity recognition and walking speed linear models. Feature extraction was performed in MATLAB (MathWorks, Natick, MA).

\section{F. Feature Selection}

1) Activity Type Recognition: Feature selection was based on correlation, following the assumption that a good feature set includes features highly correlated with the class, but uncorrelated to each other. This step, as well as the subsequent classification, was implemented in Java using libraries provided by the $W E K A$ machine learning toolkit (University of Waikato, Hamilton, New Zeland). The final feature set (see Section V-A) was used to train the SVM.

2) Multiple Linear Regression Models: Feature selection for multiple linear regression models (six activity-specific EE models, one for each cluster, the walking speed estimator and the HR normalization factor estimator) were based on how much variation in the dependent variable each feature could explain, using linear forward selection. Participant-independent models were developed for each multiple linear regression model. Additionally, anthropometrics characteristics and Resting Metabolic Rate were added to the EE models depending on the cluster [5] (see Section V-D).

\section{G. Statistics and Performance Measure}

All analysis were performed independent of the participant. Models were derived on all the participants but one, and validated on the remaining one. This leave-one-out procedure was carried out $N$ times ( $N=$ number of participants), and results were averaged. Even though performance was evaluated independent of the subject, the reported models are derived including data from al participants (Tables II, III and IV). Performance of the activity recognition was evaluated using the percentage of correctly classified instances for each cluster. The performance measures used for $\mathrm{EE}$ is the Root Mean Square Error (RMSE), averaged within an activity and between participants. Results are reported only in terms of RMSE because of the large inter-individual variability that is typical for EE estimates. Normalization procedures do exist (e.g. estimating in $\mathrm{kcal} / \mathrm{kg}$ ), but do not take into account that EE during different activities is affected differently by body weight. Performance of the walking speed linear model, as well as the HR normalization factor estimates, were evaluated using the RMSE and the percentage of the explained variance of the multiple linear regression model $\left(R^{2}\right)$. As statistical analysis, paired t-tests between non-normalized and normalized results were used. Significance level $\alpha$ was set to 0.05 for all tests.

\section{Measurement Setup and Data Collection}

\section{A. Participants}

Twenty-nine (22 male, 7 female) healthy participants took part in the experiment. Mean age was $30.9 \pm 5.5$ years, mean weight was $72.6 \pm 12.5 \mathrm{~kg}$, mean height was $177 \pm 9.3 \mathrm{~cm}$ and mean BMI was $23.0 \pm 2.6 \mathrm{~kg} / \mathrm{m}^{2}$. Our internal Ethics Committee approved the study, and each participant signed an informed consent form.

\section{B. Instruments}

1) ECG Necklace: The ECG Necklace [11] is a low power wireless ECG platform (see Fig. 4). The system relies on an ultra-low-power ASIC for ECG read-out, and it is integrated in a necklace, providing ease-of-use and comfort while allowing flexibility in lead positioning and system functionality. It achieves up to 6 days autonomy on a $175 \mathrm{mAh}$ Li-ion battery. For the current study, the ECG Necklace was configured to acquire one lead ECG data at $256 \mathrm{~Hz}$, and ACC data from a three-axial accelerometer (ADXL330) at $32 \mathrm{~Hz}$. The sensor was placed on the chest with an elastic belt. The $x$, $y$, and $z$ axes of the accelerometer were oriented along the vertical, medio-lateral, and antero-posterior directions of the body, respectively. Two gel electrodes were placed on the participant's chest, in the lead II configuration. Data were recorded on the on-board SD card to ensure integrity. Data were also streamed in real-time to provide visual feedback of the system functionality to the experimenter. 


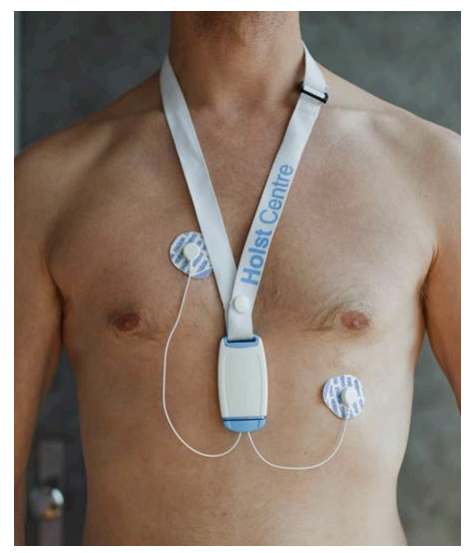

Fig. 4. Wearable sensor used for this study (ECG Necklace). The sensor acquires 2-leads ECG and 3-axial acceleration.

2) Indirect Calorimeter: Breath-by-breath data were collected using the Cosmed $K 4 b^{2}$ indirect calorimeter. The Cosmed $K 4 b^{2}$ weights $1.5 \mathrm{~kg}$, battery included, and showed to be a reliable measure of EE [12]. The system was manually calibrated before each experiment according to the manufacturer instructions. This process consists of allowing the system to warm-up, following a double calibration, first with ambient air and then with calibration gas values. A delay calibration was performed weekly to adjust for the lag time that occurs between the expiratory flow measurement and the gas analyzers.

\section{Experiment Design}

Participants were invited for recordings on two separate days. They reported at the lab at 8.00 a.m., after refraining from drinking (except for water), eating and smoking in the two hours before the experiment. The protocol included a wide range of lifestyle and sport activities, including sedentary and household activities. More specifically, day one consisted of activities selected as representative of common daily living of many people in industrialized countries [14]. The activities were: lying down, resting, sitting stretching, standing stretching, desk work, reading, writing, working on a PC, watching TV, fidgeting legs, standing still, standing preparing a salad, washing dishes, stacking groceries, folding clothes, cleaning the table, washing windows, sweeping, vacuuming, walking self-paced, walking self-paced carrying books (4.5 kg), climbing stairs up, climbing stairs down. Each sedentary and household activity was carried out for a period ranging from 4 to 12 minutes, with a 1 or 2 minutes break between the activities. Day two was carried out at the gym, where subjects performed a series of more vigorous activities, including: walking at 3,4,5 and $6 \mathrm{~km} / \mathrm{h}$ on a treadmill, walking at 4 $\mathrm{km} / \mathrm{h}$ carrying a weight (5\% of the subject's weight), walking at $3 \mathrm{~km} / \mathrm{h}, 5$ and $10 \%$ inclination, walking at $5 \mathrm{~km} / \mathrm{h}$, 5 and $10 \%$ inclination, cycle ergometer at 60 and $80 \mathrm{rpm}$, low, medium and high resistance levels, running at 7,8,9 and $10 \mathrm{~km} / \mathrm{h}$. Activities carried out at the gym were 4 minutes duration, except for free weights and running, which lasted for 1 to 2 minutes. Four participants did not perform all running activities and were excluded from data analysis.

\section{Pre-processing}

The dataset considered for this work contains about 70 hours of annotated data collected from 29 subjects, consisting of reference $\mathrm{VO}_{2}, \mathrm{VCO}_{2}$, three axial acceleration and ECG.

1) ECG Necklace Data: Raw ECG and ACC data were downloaded from the SD card of the ECG Necklace. Raw data were exported into $c s v$ files containing time-stamped ECG and acceleration samples. A Continuous Wavelet Transform based beat detection algorithm was used to extract R-R intervals from ECG data, which output was manually examined to correct for missed beats.

2) Indirect Calorimeter Data: Breath-by-breath data acquired from the Comsed $K 4 b^{2}$ was resampled at $0.5 \mathrm{~Hz}$. EE was calculated from $\mathrm{O}_{2}$ consumption and $\mathrm{CO}_{2}$ production using Weir's equation [15]. The first 1 or 2 minutes of each activity were discarded to remove non-steady-state data.

\section{RESULTS}

\section{A. Activity Recognition}

Subject-independent classification accuracy of the SVM used to select which cluster model to use in the EE estimate was $94.3 \%$. More specifically, the accuracy was $100 \%$ for lying, $91 \%$ for sedentary, $87 \%$ for dynamic, $98 \%$ for walking, $91 \%$ for biking and $99 \%$ for running.

\section{B. Walking Speed Estimator}

The walking speed multiple linear regression model could explain $94 \%$ of the variance in walking speed $\left(R^{2}=0.94\right)$. RMSE of the model is $0.28 \pm 0.09 \mathrm{~km} / \mathrm{h}$.

\section{Heart Rate Normalization Factor Estimator}

The Heart Rate Normalization Factor multiple linear regression model could explain $87 \%$ of the variance $\left(R^{2}=\right.$ 0.87). RMSE was 8.3 beats per minute (bpm) (see Fig. 5). Higher error was obtained with a second model, built using lower walking speeds only, since lower speeds will have higher chance to be detected in daily life ( 3 and $4 \mathrm{~km} / \mathrm{h}$, together with height and age, RMSE $11.8 \mathrm{bpm}$ ). Fig. 6 shows an example of the normalization. By considering our normalization factor approach, the HR variance was clearly reduced. We concluded that the normalized HR can be used as part of the activityspecific EE models, reducing over or under-estimations.
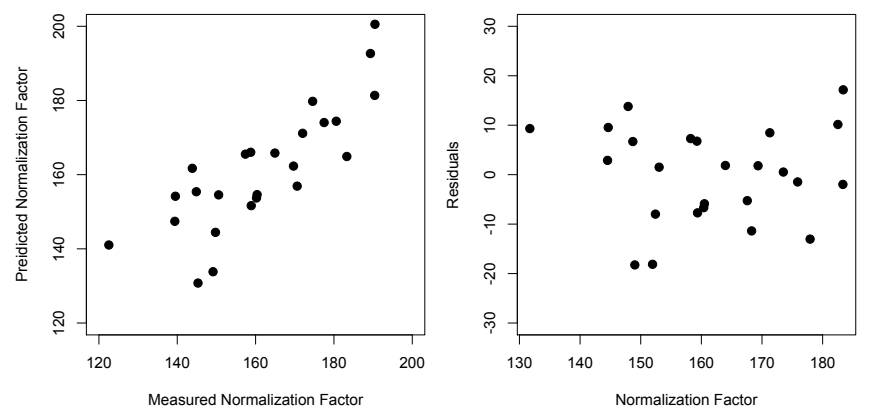

Fig. 5. Scatterplot and residuals plot of measured (running on a treadmill) VS predicted (from age, height, HR at rest and while walking at 4,5 and 6 $\mathrm{km} / \mathrm{h}$ ) normalization factors (i.e. HR while running at $10 \mathrm{~km} / \mathrm{h}$ ). 


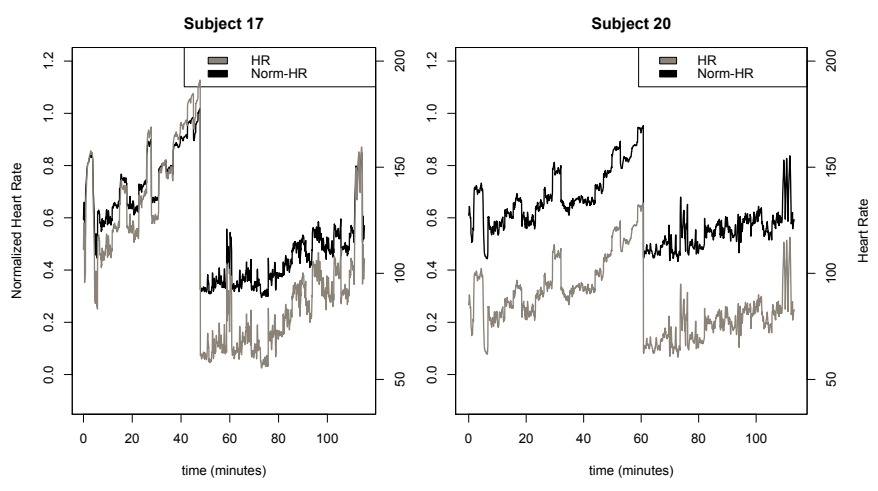

Fig. 6. HR normalized for two participants using the approach proposed in this work. Once CRF is taken into account, absolute HR differences are significantly reduced, and Norm-HR can be used to estimate EE, reducing error. HR before normalization is shown for comparison (as in Fig. 1).

\section{Energy Expenditure Estimation}

RMSE for the EE estimate was $0.60 \mathrm{kcal} / \mathrm{min}$. More specifically, RMSE was $0.20 \mathrm{kcal} / \mathrm{min}$ for lying, $0.25 \mathrm{kcal} / \mathrm{min}$ for sedentary, $0.58 \mathrm{kcal} / \mathrm{min}$ for dynamic, $0.81 \mathrm{kcal} / \mathrm{min}$ for walking, $0.92 \mathrm{kcal} / \mathrm{min}$ for biking and $0.89 \mathrm{kcal} / \mathrm{min}$ for running).

Fig. 7 shows the reduction in error for activity-specific EE models using HR (dynamic, walking, running and biking), when CRF is taken into account. RMSE was reduced from 0.60 to $0.58 \mathrm{kcal} / \mathrm{min}$ for dynamic $(3 \%$ error reduction, not significant), from 1.13 to $0.81 \mathrm{kcal} / \mathrm{min}$ for walking $(28 \%$ error reduction, $p=0.00027<\alpha$ ), from 1.38 to $0.92 \mathrm{kcal} / \mathrm{min}$ for biking ( $33 \%$ error reduction, $p=0.00037<\alpha$ ) and from 1.25 to $0.89 \mathrm{kcal} / \mathrm{min}$ for running $(29 \%$ error reduction, $p=0.01<\alpha)$.

\section{DISCUSSION AND CONCLUSIONS}

In this paper we proposed a novel algorithm for activityspecific EE estimation based on a combination of ACC and HR data. By introducing a HR normalization factor, we were able to model the effect of CRF on HR during exercise. By normalizing HR responses from subjects with different levels of CRF, we could significantly reduce EE estimation error $(p<0.05$ for walking, biking and running). More specifically, the proposed approach is able to reduce EE estimation error of activityspecific linear models (i.e. models developed specifically for an activity, and already including the best ACC features, as well as anthropometric characteristics) by an additional 28 to $33 \%$ compared to the best state-of-the-art models published up to date. The error reduction applies to non-sedentary clusters of activities, such as walking, biking or running at moderate intensities.

We believe this is a significant step towards personalized health and wellbeing monitoring. The proposed system uses a single monitoring device and is able to learn automatically from the user over time, collecting HR data while performing different activities (walking at different speeds, resting, etc.). The collected data is then used to determine the HR normalization factor, a coefficient representative of the CRF level of an individual.
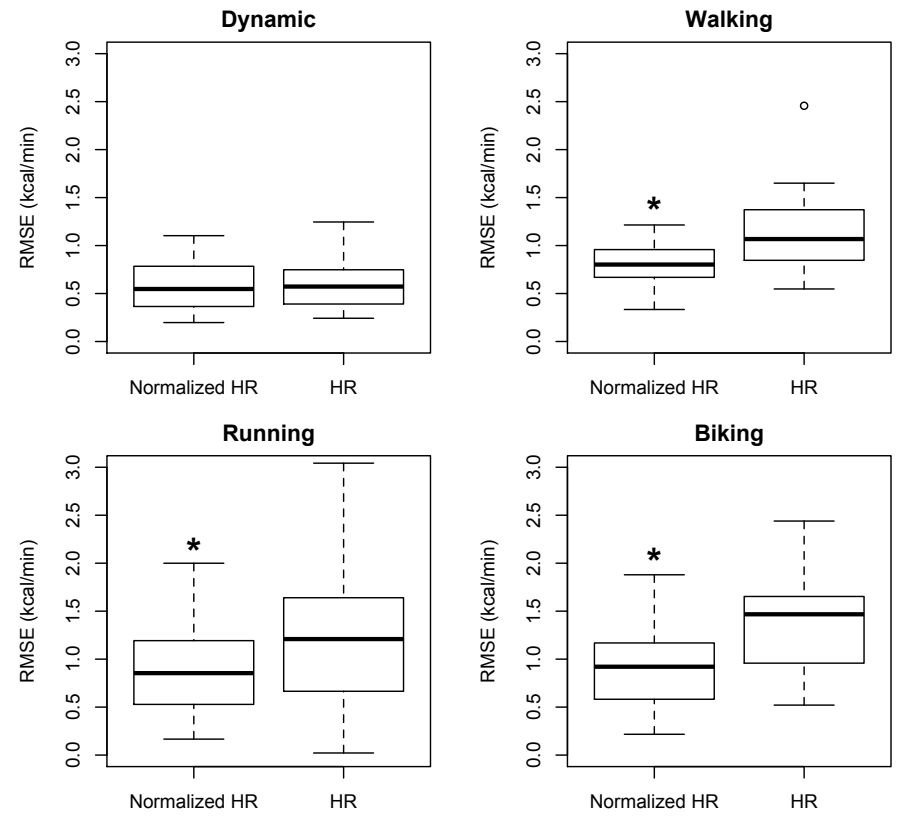

Fig. 7. RMSE for the four moderate to vigorous clusters. Statistically significant differences are marked with * (paired t-test, $p<\alpha, \alpha=0.05$ ) EE estimation error was significantly reduced for walking, running and biking. Error variance is reduced as well.

Personalizing a system goes beyond the inclusion of the individual's anthropometric characteristics in the activity-specific EE linear models. In the future, the estimated normalization factor could be used as predictor to estimate $\mathrm{VO}_{2}$ max, using equations published in literature [13]. By doing so, a user would be aware of one of the most important health markers [10], without the burden and risks of regularly performing maximal or sub maximal tests.

We expect that our HR normalization approach will be most useful for sports training devices, where users and trainers are interested in accurate EE estimation under moderate to vigorous workloads. However, less active users willing to take up a more active lifestyle, or undergoing a physical activity intervention targeted in modifying behavior to increase level of activity, would also benefit. As a matter of fact, in the latter case $\mathrm{CRF}$ takes even a bigger role, since it typically changes faster in the transition from inactive to active lifestyle, while lower changes can be expected for a continuously active lifestyle. Being able to monitor changes in CRF and HR over time would affect positively EE estimates, since EE estimates are highly dependent on HR and on the relation between CRF, HR and EE, as shown by our analysis. New opportunities for applications targeted at inducing behavioral change analyzing not only levels of PA, but also change in CRF and associated reduced risk of disease, could be developed building up on the proposed approach.

We recognize limitations in our study. Even though we developed an algorithm able to derive the HR normalization factor automatically, during regular activities, by combining rest and walking data with the subjects anthropometric characteristics, we tested it using laboratory recordings only. We consider that the evaluation with lab data is a necessary first step, which can be sufficiently covered with reference 
measurements of EE. In particular, the approach allowed us to confirm performances of the individual estimators (activity recognition accuracy was $94.3 \%$, walking speed RMSE was $0.28 \pm 0.09 \mathrm{~km} / \mathrm{h})$. Overall, we conclude that an accurate personalized EE estimation using a single monitoring device and combining ACC and HR is feasible.

\section{REFERENCES}

[1] S.J. Marshall, E. Ramirez: Reducing sedentary behavior: a new paradigm in physical activity promotion. American Journal of Lifestyle Medicine. Vol 5: No 6 (518-530); 2011.

[2] S. Brage, U. Ekelund, N. Brage, et al. Hierarchy of individual calibration levels for heart rate and accelerometry to measure physical activity. J Appl Physiol 2007;103:68292.

[3] S. Brage, N. Brage, P.W. Franks et al. Branched equation modeling of simultaneous accelerometry and heart rate monitoring improves estimate of directly measured physical activity energy expenditure. J Appl Physiol 2004;96:343351.

[4] S.M. Ceesay, A.M. Prentice, K.C. Day et al. The use of heart rate monitoring in the estimation of energy expenditure: a validation study using indirect whole body calorimetry. Br J Nutr 1989;61:175186.

[5] M. Altini, J. Penders, O. Amft. Energy Expenditure estimation using wearable sensors: a new methodology for activity-specific models. In Proceedings of Wireless Health 2012.

[6] F. Albinali, et al. Using wearable activity type detection to improve physical activity energy expenditure estimation. In UbiComp, pages 311320, 2010.

[7] M. Rumo, O. Amft, G. Troster, and U. Mader. A stepwise validation of a wearable system for estimating energy expenditure in field-based research. Physiological measurement, 32(12):19832001, 2011.

[8] A.G. Bonomi, et al. Improving assessment of daily energy expenditure by identifying types of physical activity with a single accelerometer. Journal of Applied Physiology, 107(3):655661, 2009.

[9] E. Tapia. Using machine learning for real-time activity recognition and estimation of energy expenditure. In PhD thesis, MIT, 2008.

[10] D.C. Lee, E.G. Artero, X. Sui, et al: Mortality trends in the general population: the importance of cardiorespiratory fitness. J Psychopharmacol 2010;24:27-35.

[11] J. Penders. A low-power wireless ECG necklace for reliable cardiac activity monitoring on-the-move. In EMBC 11, 2011.

[12] J.E. McLaughlin, et al. Validation of the COSMED K4 b2 portable metabolic system. International $\mathbf{J}$ of sports medicine, 22(4):280284, May 2001.

[13] J.D. George, P.R. Vehrs, P.E. Allsen, et al. Development of a submaximal treadmill jogging test for fit college-aged individuals. Med Sci Sports Exerc. $1993 ; 25: 643647$.

[14] D.R. Bassett, A. Rowlands, and S.G. Trost. Calibration and validation of wearable monitors. Med Sci Sports Exerc, 44(1 Suppl 1):S32S38, Jan. 2012.

[15] J. Weir. New methods for calculating metabolic rate with specific reference to protein metabolism. J Physiol, 109:19, 1949.

[16] M.R. Esco, E.M. Mugu, H.N. Williford, A.N. McHugh, B.E Bloomquist. Cross-Validation of the Polar Fitness Test via the Polar F11 Heart Rate Monitor in Predicting VO2Max. Journal of Exercise Physiology, 2011. 